\title{
Low Reynolds Number Turbulence Models to Simulate the Bubble Plume Behavior with the Euler-Euler Method
}

\author{
S. Besbes ${ }^{1 \dagger}$, M. El Hajem², H. Ben Aissia ${ }^{1}$ and J. Y. Champagne ${ }^{2}$ \\ ${ }^{1}$ University of Monastir, National School of Engineers of Monastir, Laboratory of Metrology and Energy \\ Systems, Monastir, 5000, Tunisia. \\ ${ }^{2}$ National Institute of Applied Science Lyon, Laboratory of Fluid Mechanics and Acoustics, 20 Av. A. \\ Einstein, 69621 Villeurbanne Cedex, France.
}

†Corresponding Author Email: soniabesbes767@gmail.com

(Received November 13, 2019; accepted June 1, 2020)

\begin{abstract}
This paper presents a comparative assessment of low Reynolds number k- $\varepsilon$ models against standard k- $\varepsilon$ model in an Eulerian framework. Three different low-Re number k- $\varepsilon$ models: Launder-Sharma (LS), YangShih (YS) and AbeKondoh-Nagano (AKN) have been used for the description of bubble plume behaviour in stratified water. The contribution of the gas phase movement into the liquid phase turbulence has been achieved by using the Dispersed with Bubble Induced Turbulence approach (DIS+BIT).The results reveal that the oscillation frequency of gas-liquid flow are correctly reproduced by standard k- $\varepsilon$ and LS models. In fact, we found for standard K- $\varepsilon$ and LS a clear dominant peak at a frequency equal to $0.1 \mathrm{~Hz}$. On the other hand, YS and AKN models have predicted chaotic oscillations. The oscillation amplitude of the bubble plume predicted from LS model seems to be in good agreement with the PIV measurements of Besbes et al. (2015). However, for the standard K- $\varepsilon$ model the oscillation amplitude is low. The air-water interface shows that the bubble plume mixing with the stratified water is predicted to be stronger compared to standard k- $\varepsilon$ model.
\end{abstract}

Keywords: Bubble plume; Computational fluid dynamics; Euler-Euler modeling; Hydrodynamics; Low Reynolds number models; Two-phase flow.

\section{INTRODUCTION}

Bubble columns are reactors with low energy consumption implementing liquid absorption from a species initially present in the gas phase by dispersing the gas phase within the liquid phase in the form of bubbles. The mass transfer at the gasliquid interface is strongly linked to the turbulence induced by the bubbles (Kawamura and Kodama, 2002; Roghair et al. 2011), to the bubble plume frequency oscillations and to the interfacial area of exchange. To understand the complex dynamics of dispersed bubbly flows computational fluid dynamics is being increasingly used, however reliable prediction depends on the application of suitable turbulence models. Furthermore, turbulence modeling is one of the main unresolved problems in the simulation of gas-liquid flows and the key to its development lies in a detailed representation of the motion of gas bubbles, and their interaction with the liquid phase (Lixing et al. 2006; Zhaoshun et al. 2002). Several alternatives have been proposed to estimate the apparent viscosity of the turbulent liquid phase in gas-liquid flows ( $\mathrm{Fu}$ et al. 2003). However, in bubble-liquid flows there are inevitably regions where the local Reynolds number of turbulence is so small that viscous effects predominate over turbulent ones. There are two methods to account for these regions in numerical computing of turbulent flows: the wall function method and the low Reynolds number modeling method. The wall function method proposed by Launder and Spalding $(1972,1974)$ has been most widely used because it economizes computer time and storage and allows the introduction of additional empirical information when the wall is rough. The low Reynolds number models have no wall functions as they compute the entire boundary layer including the viscous-sub layer and provide a damping function $f \mu$ for the eddy viscosity. Some models use $f \mu$ functions based on Ret (LaunderSharma (LS)) while others (Yang-Shih (YS) use f $\mu$ functions based on Rex (near wall treatment) and some (Abe-Kondoh-Nagano (AKN)) use a combination of both. 
In the majority of publications on numerical simulations of two-phase flows, the standard k- $\varepsilon$ model developed for single-phase flows appears to perform satisfactorily for transient simulations of gas-liquid flow (Sokolichin and Eigenberger, 1994; Sokolichin et al. 1997). The turbulence of the continuous phase in bubble columns has been investigated by Ekambara and Dhotre, 2010 to assess the performance and applicability of different turbulence models namely standard k- $\varepsilon$, RNG k- $\varepsilon$, Reynolds stress model (RSM) and large eddy simulation (LES). Recently, direct numerical simulations (DNS) data of dispersed turbulent bubbly flows have become available. This data can be used as a basis to test the model assumptions of Euler-Euler RANS. Experimentally, it is difficult to obtain the terms in the turbulent kinetic energy equation of the liquid phase. However, DNS, on sufficiently fine grids, can provide such data. Otherwise, the data can also be used to develop more elaborate closing approximations for bubble induced turbulence (BIT) terms. Several works of this type have been accomplished (Santarelli et al. 2016; Ma et al. 2017, 2020; Du Cluzeau et al. 2019) to develop a complete BIT closure in the framework of Euler-Euler two-equation RANS modelling.

The review of literature indicates that the low-Re number models are not fully explored for the description of flow pattern in bubble columns. However, these models have been widely used to predict the flow behavior of steady/unsteady singlephase flows (Patel et al. 1985; Cotton and Jackson, 1990; Hrenya et al. 1995; Chang et al. 1995; Mathur and He, 2013; Rathore and Das, 2013; Gorji et al. 2014).

This paper presents a comparative study of the performance and applicability of low-Re number k$\varepsilon$ models: Launder-Sharma (LS), Yang-Shih (YS) and Abe-Kondoh-Nagano (AKN) against the standard $\mathrm{k}-\varepsilon$ model, which is a high-Reynolds number model. An attempt was made to predict the detailed flow characteristics of gas-liquid flow in bubble column operating at low gas flow rates in homogenous regime, using an Eulerian-Eulerian formulation for the two-phase flow. For this purpose, a three-dimensional unsteady computational model elaborated with the aid of the software ANSYS Fluent was performed and the results were compared with experimental data of Besbes et al. 2015.

\section{Computational Model}

\subsection{Hydrodynamic Model}

The numerical simulations presented are based on the two-fluid Euler-Euler approach, which is based on ensemble averaged mass and momentum transport equations for each phase. The two phases are treated mathematically as interpenetrating continua, the mass and momentum conservation equations of which, without mass transfer, can be written as follows (over bars that are frequently used to indicate ensemble averaged quantities are not used, for simplicity):

$$
\frac{\partial}{\partial t}\left(\alpha_{q} \rho_{q}\right)+\nabla \cdot\left(\alpha_{q} \rho_{q} U_{q}\right)=0
$$

$\frac{\partial}{\partial \mathrm{t}}\left(\alpha_{\mathrm{q}} \rho_{\mathrm{q}} \mathrm{U}_{\mathrm{q}}\right)+\nabla \cdot\left(\alpha_{\mathrm{q}} \rho_{\mathrm{q}} \mathrm{U}_{\mathrm{q}} \mathrm{U}_{\mathrm{q}}\right)$

In the above equations, $\mathrm{q}=\mathrm{g}$ or 1 represents the gas or liquid phase, $\alpha_{\mathrm{q}}$, $\rho_{\mathrm{q}}$ respectively denote the volume fraction and density, $\mathrm{Uq}$ is the local velocity, $\mathrm{P}$ is the pressure, $\tau_{\mathrm{q}}$ is the Reynolds-averaged viscous stress tensor, $\tau_{\mathrm{Re}, \mathrm{q}}$ is the Reynolds stress tensor, $\mathrm{g}$ is the gravity constant and $\mathrm{F}_{\mathrm{qm}}(\mathrm{m}=\mathrm{g}, \mathrm{l})$ accounts for the interfacial forces that include the drag force $F_{D}$, lift force $F_{L}$, virtual mass force $F_{V M}$, wall force $F_{W L}$ and turbulent dispersion force $\mathrm{F}_{\mathrm{TD}}$ (for the RANS approach).

\subsection{Interfacial Momentum Transfer}

All Closure relations are required for estimating the interfacial momentum transfer and the Reynolds stress tensor. Lastly, assumptions have to be made regarding bubble size, since this parameter has an impact on turbulence modeling and on the interfacial interaction forces. Since we operated in the homogenous regime, which is characterized by low gas velocities $(\mathrm{Vg}=0.08 \mathrm{~cm} / \mathrm{s})$ and small spherical bubbles, relatively simple mathematical model can be capable of providing a good representation of the flow field, even in the sparger region (Silva et al. 2012). Indeed, in the case of dilute flows with spherical particles, it is possible to simplify the general particle momentum equation and consider only the gravity-buoyancy, pressure, drag and virtual mass forces. Furthermore, the drag force is the predominant interfacial interaction force with a significant higher magnitude than virtual mass, lift and turbulent dispersion forces which represents the dispersion of particles due to transport by turbulent fluid motion (Simonin, 1990). Mandar et al. (2008) have found, at low superficial gas velocity $(\mathrm{Vg}=1.2 \mathrm{~cm} / \mathrm{s})$, that the lift and turbulent dispersion forces have not significant effect on the flow pattern in comparison with high superficial velocities of different bubble sizes. Further, the virtual mass force has no significant effect on results. Since the Superficial gas velocity in this study is very low $(\mathrm{Vg}=0.08 \mathrm{~cm} / \mathrm{s})$ we have neglected the effect of lift and turbulent dispersion forces. Thus, the inter-phase momentum transfer between gas and liquid due to drag force is given by:

$\mathrm{F}_{\mathrm{D}}=\mathrm{K}_{\mathrm{gl}}\left(\mathrm{U}_{\mathrm{g}}-\mathrm{U}_{1}\right)$

Where $K_{g l}$ is the exchange coefficient and can be expressed as follows:

$\mathrm{K}_{\mathrm{gl}}=\frac{3}{4} \mathrm{C}_{\mathrm{D}} \frac{\alpha_{\mathrm{g}} \alpha_{1} \rho_{1}}{\mathrm{~d}_{\mathrm{g}}}\left|\mathrm{U}_{\mathrm{g}}-\mathrm{U}_{1}\right|$

Where $C_{D}$ and $d g$ represent the drag coefficient and the gas bubble diameter, respectively. The drag model 
employed has a significant effect on the aerated flow, as it is related directly to the bubble size and terminal velocity. The model of Morsi and Alexander, 1972 is employed in this work, where the drag coefficient is expressed as a function of the bubble Reynolds number:

$$
\begin{aligned}
& C_{D}=a_{1}+\frac{a_{2}}{\operatorname{Re}}+\frac{a_{3}}{\operatorname{Re}^{2}} \\
& \operatorname{Re}=\frac{\rho_{1}\left|U_{g}-U_{1}\right| d_{g}}{\mu_{1}}
\end{aligned}
$$

Here $\mathrm{a}_{1}=0.3664 ; \mathrm{a}_{2}=98.33$ and $\mathrm{a} 3=-2778$ are constants that apply to smooth spherical particles for $100<\operatorname{Re}<1000$.

\subsection{Turbulence Models}

The turbulence in the continuous phase was modeled using k- $\varepsilon$ model supplemented with extra terms that include the inter-phase momentum transfer while that in the dispersed phase was modeled using Tchen's theory of dispersion of discrete particles by homogeneous turbulence (Hinze, 1975). To account for two-way turbulence coupling we used the dispersed k- $\varepsilon$ model, which is suitable when the secondary phase is dilute and the primary phase is clearly continuous. This model accounts only for turbulence in the continuous phase and neglects the Reynolds stress tensor for the dispersed phase. Closure relations for the Reynolds stress tensor of the liquid phase are provided by the $\mathrm{k}-\varepsilon$ model as:

$$
\begin{aligned}
\tau_{\mathrm{Re}, \mathrm{l}}=\rho_{\mathrm{l}} \overline{\mathrm{u}_{1}^{\prime} \mathrm{u}_{1}^{\prime}}= & -\frac{2}{3}\left(\rho_{1} \mathrm{k}_{1}+\rho_{1} \mu_{\mathrm{t}, 1} \nabla \cdot \mathrm{U}_{1}\right) \tau_{1} \\
& +\rho_{1} \mu_{\mathrm{t}, 1}\left(\nabla \mathrm{U}_{1}+\nabla \mathrm{U}_{1}^{\mathrm{T}}\right)
\end{aligned}
$$

where $U_{1}$ is the Reynolds-averaged velocity, the turbulent viscosity for the liquid phase is written as:

$\mu_{\mathrm{t}, 1}=\rho_{1} \mathrm{C}_{\mu} \mathrm{f}_{\mu} \frac{\mathrm{k}_{1}^{2}}{\varepsilon_{1}}$

The general form of the transport equations for the turbulent kinetic energy and turbulent dissipation rate for the continuous phase are obtained from the modified k- $\varepsilon$ model:

$$
\begin{array}{r}
\frac{\partial}{\partial \mathrm{t}}\left(\alpha_{1} \rho_{1} \mathrm{k}_{1}\right)+\nabla \cdot\left(\alpha_{1} \rho_{1} \mathrm{U}_{1} \mathrm{k}_{1}\right)=\nabla \cdot\left(\alpha_{1} \frac{\mu_{\mathrm{t}, \mathrm{l}}}{\sigma_{\mathrm{k}}} \nabla \mathrm{k}_{1}\right) \\
+\alpha_{1} \mathrm{G}_{\mathrm{k}, \mathrm{l}}-\alpha_{1} \rho_{1} \tilde{\varepsilon}_{1}+\alpha_{1} \rho_{1} \Pi_{\mathrm{k}, 1} \\
\frac{\partial}{\partial \mathrm{t}}\left(\alpha_{1} \rho_{1} \varepsilon_{1}\right)+\nabla \cdot\left(\alpha_{1} \rho_{1} \mathrm{U}_{1} \varepsilon_{1}\right)=\nabla \cdot\left(\alpha_{1} \frac{\mu_{\mathrm{t}, 1}}{\sigma_{\varepsilon}} \nabla \varepsilon_{1}\right) \\
+\alpha_{1} \frac{\varepsilon_{1}}{\mathrm{k}_{1}}\left(\mathrm{C}_{1 \varepsilon} \mathrm{f}_{1} \mathrm{G}_{\mathrm{k}, 1}-\mathrm{C}_{2 \varepsilon} \mathrm{f}_{2} \rho_{1} \varepsilon_{1}\right)+\alpha_{1} \rho_{1} \mathrm{E}+\alpha_{1} \rho_{1} \prod_{\varepsilon, 1}
\end{array}
$$

Where $\mathrm{G}_{k, l}$ is the production of turbulent kinetic energy and it has a similar form to the one applied for single phase flow. $\tilde{\varepsilon}_{1}=\varepsilon_{1}-\mathrm{D}$ is a modified dissipation rate of $k_{l}$. The damping functions $f_{\mu}, f_{l}$ and $f_{2}$ and the extra source terms D and E are only active close to solid walls and allow to solve $k_{l}$ and $\varepsilon$ down to the viscous sub layer. Please note that the expressions provided above for turbulence modelling (Eqs. (8-10)) are general in so far that they can represent any of the above-mentioned four different turbulence models by an adequate choice of the corresponding terms, as summarized in Tables 1- 3 . The model constants, dumping functions and boundary conditions are presented in Tables 1- 3 .

Table 1 Models constants

\begin{tabular}{|c|c|c|c|c|c|}
\hline k- $\varepsilon$ model & $\mathrm{C}_{\mu}$ & $\mathrm{C}_{1 \varepsilon}$ & $\mathrm{C}_{2 \varepsilon}$ & $\sigma_{\mathrm{k}}$ & $\sigma_{\varepsilon}$ \\
\hline Standard & 0.09 & 1.44 & 1.92 & 1.0 & 1.3 \\
\hline LS & 0.09 & 1.44 & 1.92 & 1.0 & 1.3 \\
\hline YS & 0.09 & 1.44 & 1.92 & 1.0 & 1.3 \\
\hline AKN & 0.09 & 1.50 & 1.90 & 1.4 & 1.4 \\
\hline
\end{tabular}

The ability to dissipate and generate turbulence due to relative velocity between the phases (turbulent two-way coupling) was taken in to account by incorporation of additional source terms for transport equations of kinetic energy and its dissipation rate (Simonin and Viollet, 1990).

The turbulence effects on the dispersed phase are achieved by an extension of Tchen's theory (Simonin, 1990).

\subsection{Numerical Details}

The dimensions of the simulated column $267 \mathrm{~mm}$ (width) x $600 \mathrm{~mm}$ (height) $\times 15 \mathrm{~mm}$ (depth) are the same as the experimental apparatus of Besbes et al. (2015). The lateral dimensions in the horizontal direction exceed 3-4 times the initial bubble diameter. Therefore, the terminal rise velocities were not affected by the size of the computational domain (Van Sint Annaland et al. 2005).The column was initially filled with water up to a height of $\mathrm{H}_{\mathrm{L}}=500 \mathrm{~mm}$. To select a proper grid size, we simulate the problem on different meshes of which we will increase the number of cells by decreasing the step $\Delta \mathrm{x}$ and $\Delta \mathrm{y}$. A non-uniform grid was used to guarantee that the smaller control volumes are presented where variables are steeper. The mesh sizes are in geometric progression ratio of 0.95 in the horizontal direction with mesh concentration towards the column walls and are in symmetrical geometric progression in the axial direction. We have found that the refinement of the grid size from 9300 (coarse) to 18600 (medium) produces a change in the axial velocity profiles. Whereas the grid sizes of 18600 and 36600 (fine) gives practically the same results. Based on the considerations of accuracy and computational time, the grid size of 18600 for which the results are grid independent was employed in this work.

Starting at $\mathrm{t}=0.0 \mathrm{~s}$ gas was fed to the column through a needle of $0.4 \mathrm{~mm}$ diameter, numerically the needle orifice was modeled as a velocity inlet. It has been assumed that all the bubbles are spherical and for a flow rate of $0.2 \mathrm{l} / \mathrm{min}$ their average diameter is equal to $2.5 \mathrm{~mm}$ (experimental conditions of Besbes et al. 2015). No slip boundary 
Table 2 Functions in the turbulence models

\begin{tabular}{|c|c|c|c|}
\hline $\begin{array}{c}\mathrm{k}-\varepsilon \\
\text { model }\end{array}$ & $f_{\mu}$ & $f_{1}$ & $f_{2}$ \\
\hline Standard & 1 & 1 & 1 \\
\hline $\mathrm{LS}$ & $\exp \left(\frac{-3.4}{\left(1+\mathrm{Re}_{t} / 50\right)^{2}}\right)$ & 1 & $1-0.3 \exp \left(-\mathrm{Re}_{t}\right)^{2}$ \\
\hline $\mathrm{YS}$ & $\left(1+\frac{1}{\sqrt{\operatorname{Re}_{t}}}\right)\left[1-\exp \left(-a \operatorname{Re}_{x}-b \operatorname{Re}_{x}^{3}-c \operatorname{Re}_{x}^{3}\right]\right.$ & $\frac{\sqrt{R e_{t}}}{1+\sqrt{R e_{t}}}$ & $\frac{\sqrt{\operatorname{Re}_{t}}}{1+\sqrt{\operatorname{Re}_{t}}}$ \\
$\mathrm{AKN}$ & $\left(1+\frac{5}{\mathrm{Re}_{t}^{0.75}} \exp \left(-\left(\frac{\mathrm{Re}_{t}}{200}\right)^{2}\right)\left(1-\exp \left(-\frac{\mathrm{Re}_{x}}{14}\right)\right)^{2}\right.$ & 1 & $\left(1-0.3 \exp \left(-\frac{\operatorname{Re}_{t}}{6.5}\right)^{2}\right)\left(1-\exp \left(-\frac{\operatorname{Re}_{x}}{3.1}\right)\right)^{2}$ \\
\hline
\end{tabular}

Note: $\operatorname{Re}_{\mathrm{t}}=\frac{\rho_{\mathrm{l}} \mathrm{k}_{1}^{2}}{\mu_{1} \varepsilon_{1}} ; \operatorname{Re}_{\mathrm{x}}=\frac{\mathrm{x} \rho_{\mathrm{l}} \sqrt{\mathrm{k}_{1}}}{\mu_{1}}$

Table $3 \mathrm{D}$ and $\mathrm{E}$ terms along with the boundary conditions

\begin{tabular}{|c|c|c|c|}
\hline $\mathrm{k}-\varepsilon$ model & $\mathrm{D}$ & $\mathrm{E}$ & Wall BC \\
\hline standard & 0 & 0 & $\frac{\partial k_{l}}{\partial x}=0$ \\
\hline $\mathrm{LS}$ & $2 \frac{\mu_{l}}{\rho_{l}}\left(\frac{\partial \sqrt{k_{l}}}{\partial x}\right)^{2}$ & $2 \frac{\mu_{l} \mu_{t, l}}{\rho_{l}}\left(\frac{\partial^{2} U_{i}}{\partial x^{2}}\right)^{2}$ & $k_{l}=0 ; \widetilde{\varepsilon}_{l}=0$ \\
\hline $\mathrm{YS}$ & 0 & $\frac{\mu_{l} \mu_{t, l}}{\rho_{l}}\left(\frac{\partial^{2} U_{i}}{\partial x^{2}}\right)^{2}$ & $k_{l}=0 ; \varepsilon_{l}=\frac{\mu_{l}}{\rho_{l}}\left(\frac{\partial^{2} k_{l}}{\partial x^{2}}\right)$ \\
\hline $\mathrm{AKN}$ & 0 & 0 & $k_{l}=0 ; \varepsilon_{l}=\frac{\mu_{l}}{\rho_{I}}\left(\frac{\partial \sqrt{k_{l}}}{\partial x}\right)^{2}$ \\
\hline
\end{tabular}

Note: $\mathrm{x}$ local coordinate normal to the wall

conditions were used at all the impermeable walls

for both the continuous phase and the dispersed phase. The top surface of the column was specified as a pressure outlet where the atmospheric pressure is imposed on the top of the column.

The resulting set of Equations for the liquid phase was solved using the commercial solver Fluent based on a finite volume method. The phase coupled simple algorithm was used for the pressure velocity coupling in the momentum equation. The time derivatives were discretized using a second order implicit method, while the diffusive and convective terms for velocities, volume fraction, kinetic energy $(\mathrm{k})$ and its dissipation rate $(\varepsilon)$ were discretized using the higher-order Quick discretization scheme. An Eulerian time step of $10^{-2}$ $\mathrm{s}$ was used in all simulations except for the case with virtual mass force the time step was refined to $5 \times 10^{-3} \mathrm{~s}$. The convergence criterion $1 \times 10^{-5}$ was used in the present work.

\section{RESULTS AND DISCUSSION}

The dynamic flow structure, which consists of several large-scale vortices, continuously changing their location in time at low frequencies, governs the mixing process of both phases. The realistic description of these large-scale dynamics is therefore an important requirement for the proper modeling of gas-liquid reactors. Simulations are used to assess the performance of low Reynolds number turbulence models. For this purpose, standard $\mathrm{k}-\varepsilon$ model is used to reproduce and compare with simulations of low Reynolds number turbulence models: LS, YS and AKN. In addition, simulations are compared with our previous PIV measurements (Besbes et al. 2015).

\subsection{Assessment of Low-Re Number Models against Standard K- $\varepsilon$ Model}

The turbulent predictions of the dispersed phase are 


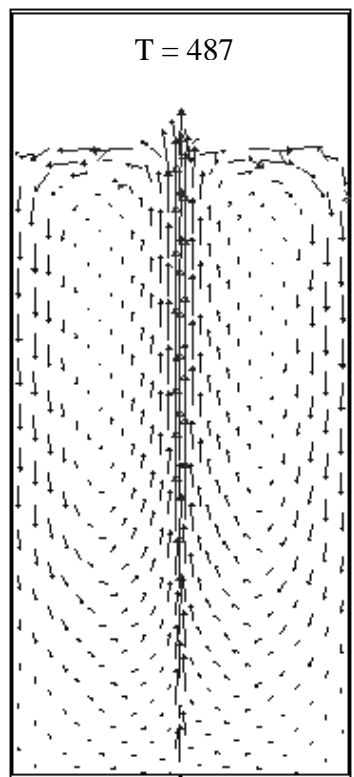

Fig. 1. Standard $k-\varepsilon$ simulations of mean liquid velocity vectors at the mid-depth plane of the column.

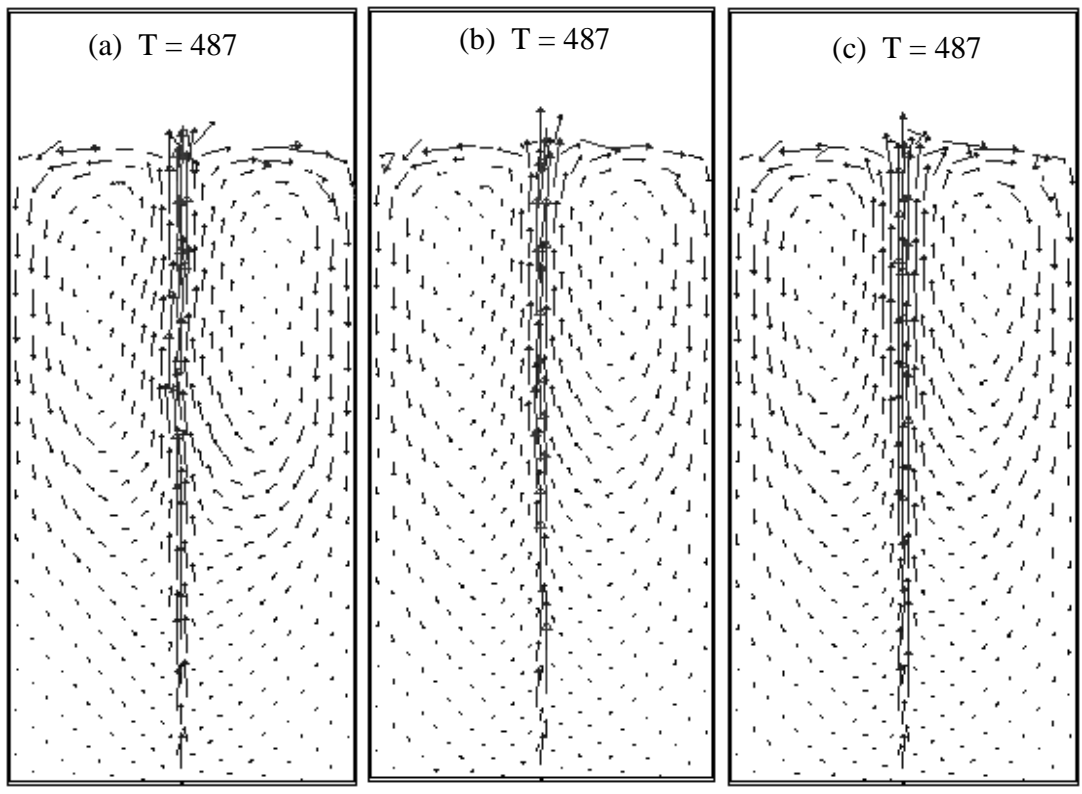

Fig. 2. Low Reynolds number turbulence simulations of mean liquid velocity vectors (a) LS, (b) YS and (c) AKN.

achieved by using the Dispersed with Bubble Induced Turbulence approach (DIS+BIT : $C_{3 \varepsilon}=1.2$ ) which takes into account the contribution of the gas phase movement into the liquid phase turbulence. The snapshot of the mean liquid velocity vectors at the mid depth plane of the column are represented in Figs. 1 and 2. The time averaged flow regime characterized by a strong upflow in the central part of the column and downward flow in the wall region can be observed. From this flow field, two separate circulation cells can be seen to dominate the flow pattern, which still resembles the "cooling tower" mode of circulation, despite the dynamic behavior of the bubble plume
(Fig. 1). These "cooling tower" flow pattern still prevails for the predictions of low Reynolds number turbulence models (LS, YS and AKN) as can be derived from Fig. 2.

In view of unavailability of transient experimental data at spatial locations, long time average is taken and compared with the experimental data. The performance of all models can be discussed in context of Figs. 3 and 4 which show the comparison of computed and measured averaged vertical liquid velocity profiles at two positions above the needle $\mathrm{Y}^{*}=0.25$ (Fig. 3) and 0.75 (Fig. 4). Here $\mathrm{Y}^{*}$ is a dimensionless axial coordinate with respect to the liquid height $\left(\mathrm{Y}^{*}=\mathrm{Y} / \mathrm{H}_{\mathrm{L}}\right)$ and $\mathrm{X}^{*}$ is dimensioned 


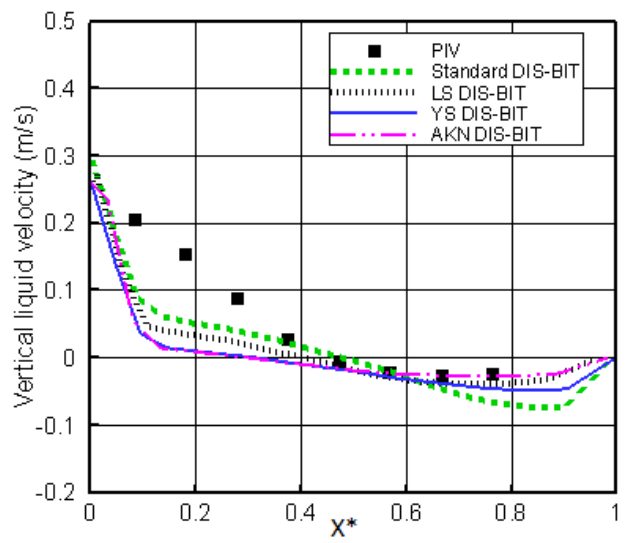

Fig. 3. Time-averaged vertical liquid velocity profiles for $Q=0.2 \mathrm{l} / \mathrm{min}$, at $Y^{*}=0.25$.

with respect to the column width $(\mathrm{W})\left(\mathrm{X}^{*}=\mathrm{X} / \mathrm{W}\right)$. As can be seen from these figures, for all models there is a discrepancy between the numerical results and experiment near the core region showing an enlargement of the rising liquid area which was not well reproduced by the simulations. In fact, all models cannot reproduce the radial dispersion in the gas phase and the spread of the bubble plume is much smaller than that observed experimentally. This shows that the different size vortices of the computed flow field do not disperse the bubble flow sufficiently. Instead the dispersion is caused by numerous small vortices and flow variations caused by the liquid flow around individual bubbles or bubble clusters. So, all models cannot resolve these small scale phenomena. We can observe from Fig. 3 , at $\mathrm{Y}^{*}=0.25$ (near the wall region) that, LS, YS and $\mathrm{AKN}$ models, as a modified version of standard $\mathrm{K}-\varepsilon$ model by the inclusion of damping functions in order to account for the viscous and wall effects, reveal satisfying agreement with the experimental data better than the standard K- $\varepsilon$ model. Reason for the better agreement is a more realistic value of the eddy viscosity of the liquid phase. However, at the core region the standard $\mathrm{k}-\varepsilon$ model performs better than the low Reynolds number models. On the other hand, at $\mathrm{Y}^{*}=0.75$, Fig. 4 shows that, at the core region the LS model shows the best prediction of experimental results.

\subsection{Transient Phenomena}

The calculation of the power spectra should allow characterizing some important features of the typical flow regimes taking place in a bubble column and determining the existence of periodic structures and yield their specific frequencies. The vertical velocity signal and the power spectra at point $\left(\mathrm{X}^{*}=0, \mathrm{Y}^{*}=0.5\right)$, for different turbulence models are shown in Fig. 5 at the mid-depth plane of the column. We can identify for standard K- $\varepsilon$ and LS a clear dominant peak at a frequency equal to $0.1 \mathrm{~Hz}$, which puts into evidence the appearance of liquid macrostructures, and can be related to the experimentally observed oscillation of a central plume of bubbles (Besbes et al. 2015). However,

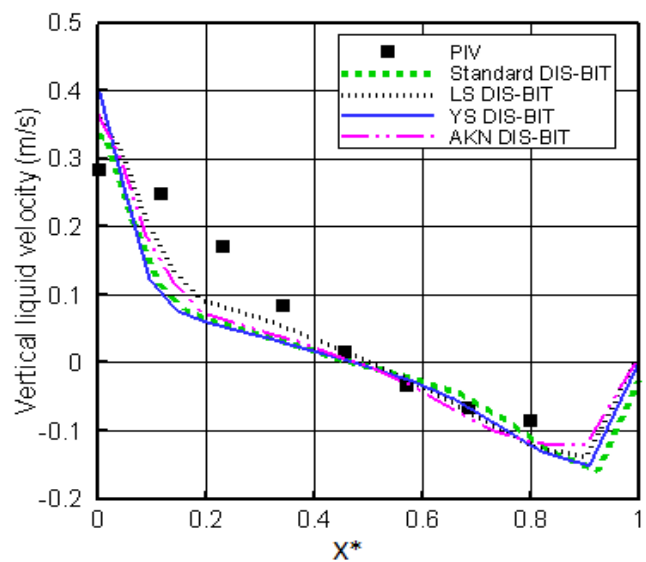

Fig. 4. Time-averaged vertical liquid velocity profiles for $Q=0.2 \mathrm{l} / \mathrm{min}$, at $Y^{*}=0.75$.

for LS model a second peak at a frequency of 0.2 $\mathrm{Hz}$ can be distinguished, corresponding to the destruction of the preceding structure, also observed by Chen et al. 1994 and indicating the strong unstationarity of the system. In addition, quantitative agreement of the evolution of the vertical velocity between standard $\mathrm{K}-\varepsilon$ and LS models can be observed until $50 \mathrm{~s}$, after that the solutions tend to deviate more and more from each other as time proceeds. On the other hand, Fig. 5 shows chaotic oscillations of vertical liquid velocity profiles predicted by the YS and AKN models. From the above results, besides the standard K- $\varepsilon$ model only the LS model can reproduce the oscillatory movement of the bubble plume. Thus, a qualitative comparison of the instantaneous PIV images of the oscillating bubble plume from Besbes et al. 2015 and from numerical simulations of standard K- $\varepsilon$ and LS models is represented in Figs. 6 and 7. As seen from Fig. 6, the direction of the lower part of the bubble plume is stable however, the upper part changes its appearance and location corresponding to the transient liquid circulation flows. Figure 7 shows that the oscillation amplitude of the bubble plume is low for the standard K- $\varepsilon$ model. However, for LS model, the oscillation amplitude is in accordance with the results of Besbes et al. 2015. This can be attributed to a more realistic value of the eddy viscosity of liquid phase for LS model. In addition, the plume seems to be wider from LS model; however, for standard K- $\varepsilon$ model, the bubble plume width is lower.

Indeed, the dynamic behavior of the air-water interface has an impact on the oscillating bubble plume. So, to analyze the free surface dynamics induced by the rising bubbles a close-up of the time averaged air-water interface predicted from standard $\mathrm{K}-\varepsilon$ and LS simulations is represented in Fig. 8. This figure shows that for LS model air bubble plume mix with the stratified water largely. However, future work from this study should be done to dig in to the enlargement and the behavior of the oscillating bubbles plume. 
S. Besbes et al. / JAFM, Vol. 14, No. 1, pp. 1-10, 2021.
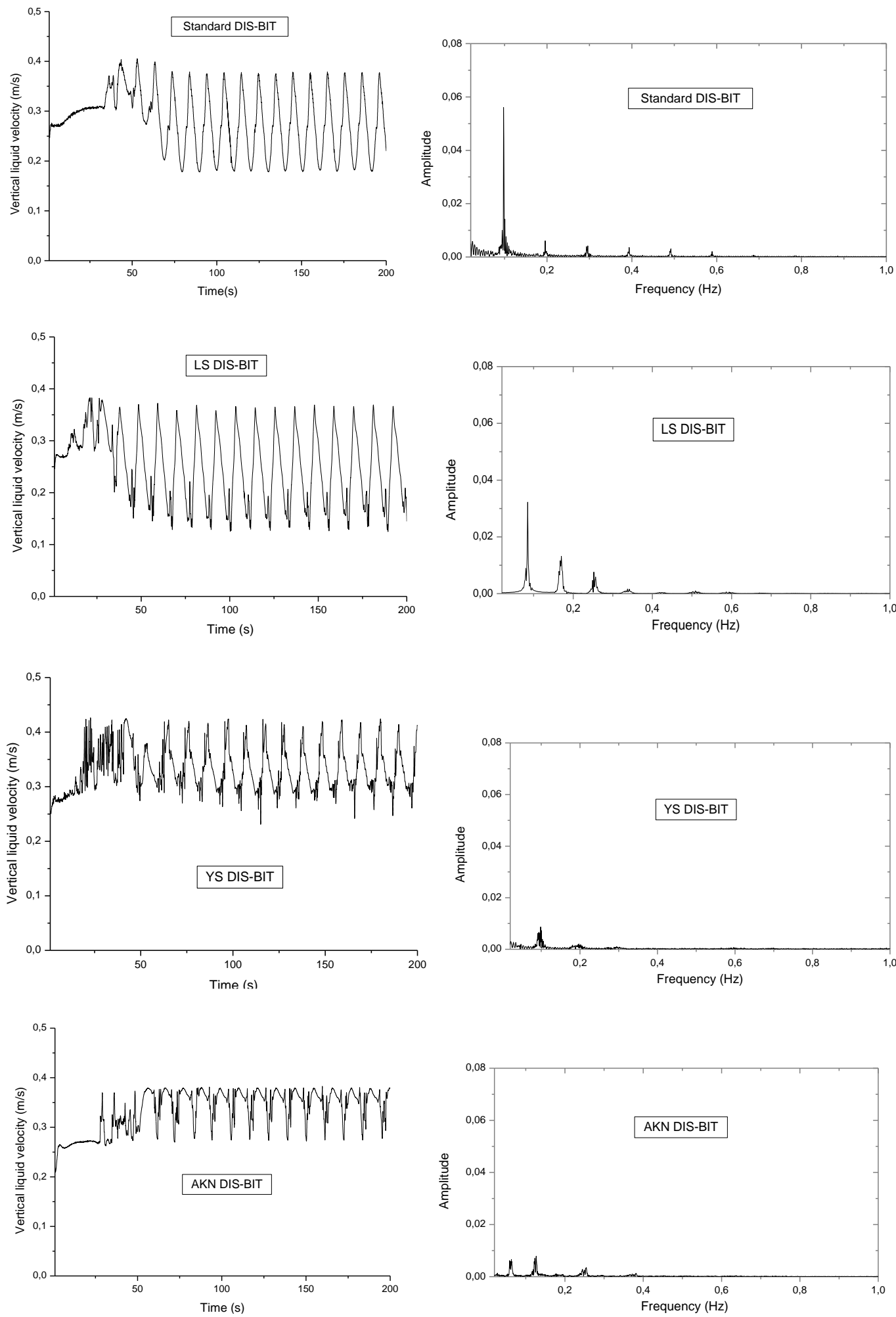

Fig. 5. Simulations of time series of vertical liquid velocities and corresponding spectral density, at gas flow rate $Q=0.2 \mathrm{l} / \mathrm{min}$, at point $\left(X^{*}=0, Y^{*}=0.5\right)$ and at the mid-depth plane of the column.

\section{CONCLUSION}

The performance of three low Reynolds number k- $\varepsilon$ models in predicting unsteady dynamic characteristics of the oscillating bubble plume in a needle sparger rectangular bubble column is evaluated against standard $\mathrm{k}-\varepsilon$ model. The contribution of the gas phase movement into the 


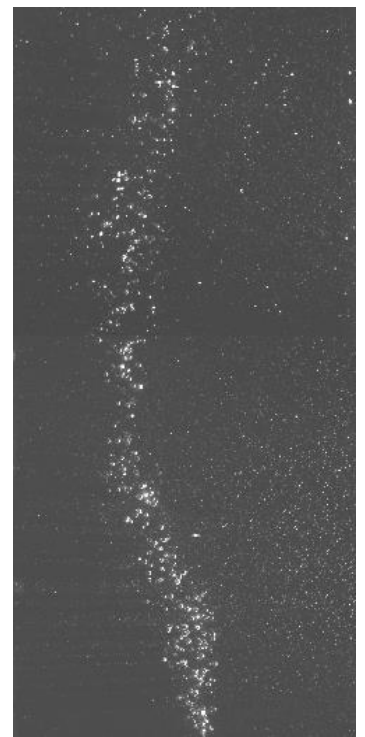

Fig.6. PIV images of oscillating bubble plume at gas flow rate $Q=0.21 / \mathrm{mn}$ (Besbes et al. 2015).
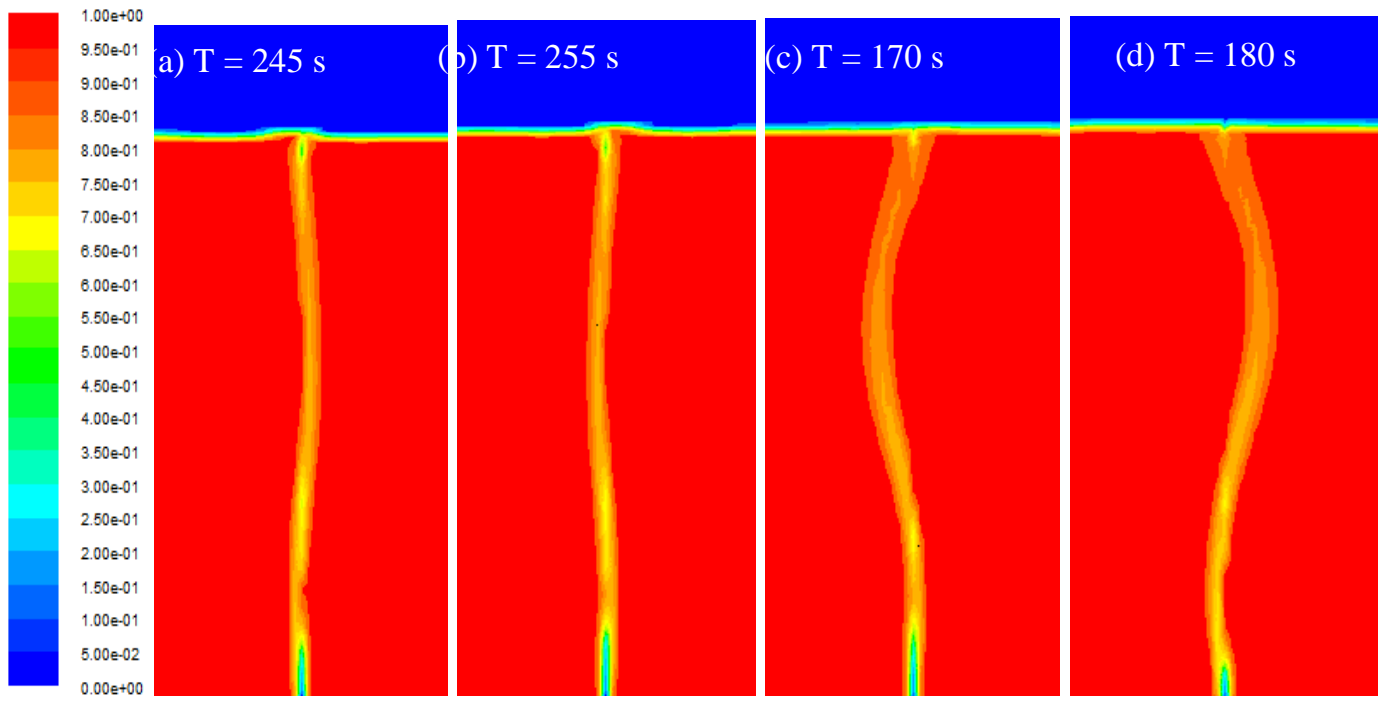

Fig. 7. Simulations of bubble plume oscillations: (a,b) Standard DIS-BIT; (c,d) LS DIS-BIT.

liquid phase turbulence (DIS+BIT) has been considered. The cooling tower flow pattern characterized by a strong up-flow in the central part of the column and downward flow in the wall region still prevails for the predictions of the low Reynolds number turbulence models. However, no models can reproduce the radial dispersion in the gaz phase and the spread of the bubble plume is much smaller than that observed experimentally. This shows that the different size vortices of the computed flow field do not disperse the bubble flow sufficiently.

We have found that near the wall region, at $\mathrm{Y}^{*}=$ 0.25 , LS, YS and AKN models as a modified version of standard K- $\varepsilon$ model by the inclusion of damping functions in order to account for the viscous and wall effects reveal satisfying agreement with the experimental data of Besbes et al. 2015 slightly better than the standard K- $\varepsilon$ model.
However, at the core region the standard k- $\varepsilon$ model performs the best. Near the column top $\left(\mathrm{Y}^{*}=0.75\right)$, at the core region the LS model shows the best prediction of experimental results. So, none of the low Reynolds number $k-\varepsilon$ models tested are capable of predicting the local liquid velocity profiles in good agreement with experimental results over the hole domain.

The analysis of the power spectra for different turbulence models shows a clear dominant peak at a frequency equal to $0.1 \mathrm{~Hz}$ for standard $\mathrm{k}-\varepsilon$ and LS, which is close to the value obtained by Besbes et al. 2015. However, LS model shows a second peak at a frequency of $0.2 \mathrm{~Hz}$. Thus, the detailed characteristics of gas-liquid flow are correctly reproduced by the standard K- $\varepsilon$ and LS models. On the other hand, the oscillation amplitude of the bubble plume predicted from LS model seems to be qualitatively in good agreement with PIV 


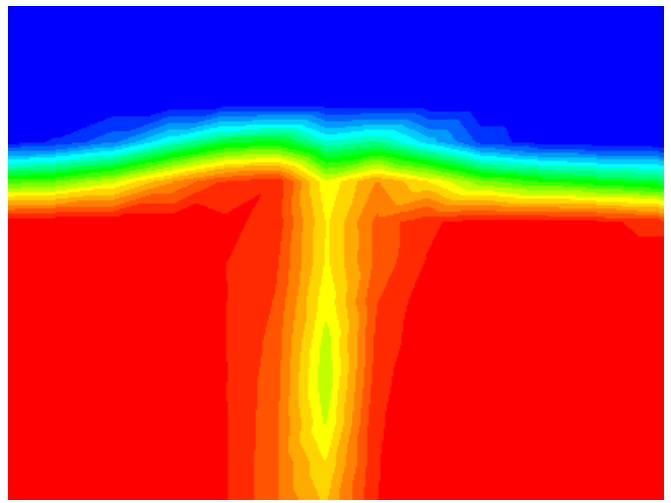

(a) Mean flow at $\mathrm{T}=492 \mathrm{~s}$

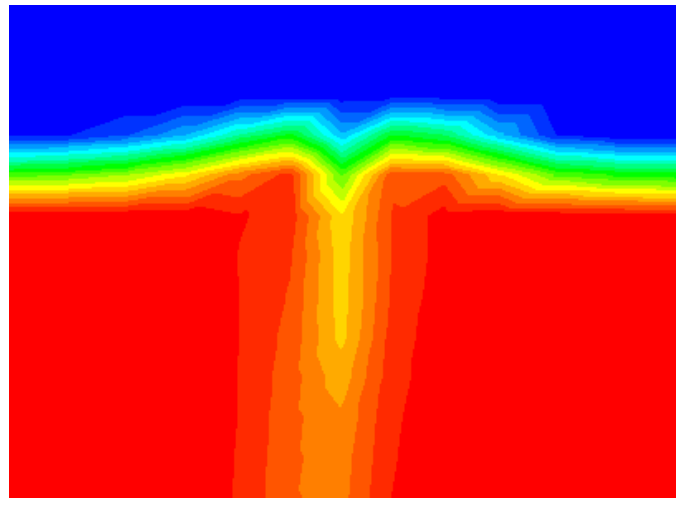

(b) Mean flow at $\mathrm{T}=260 \mathrm{~s}$

Fig. 8. Close up of air-water interface: (a) Standard DIS-BIT; (b) LS DIS-BIT.

measurements of Besbes et al. 2015. This may be related to a more realistic value of the eddy viscosity of liquid phase for LS model.

Besides standard $\mathrm{k}-\varepsilon$ model, among the lowReynolds turbulence models examined, only the LS model can reproduce the oscillatory movement of the bubble plume, through an appropriately designed damping function. However, the comparison of the performance of the low-Reynolds turbulence models should be validated against more detailed turbulent parameter measurements such as Reynolds stress. Therefore, at this stage of comparison, limited to a liquid velocity profiles, we cannot say that the LS model is the only suitable model for such unsteady flows. This work will be pursued in future, if a larger number of detailed experimental measurements are available.

The time averaged air-water interface predicted from standard K- $\varepsilon$ and LS simulations shows that for LS model air bubble plume mix with the stratified water largely. Consequently, the bubble plume from LS simulations seems to be wider than that from standard K- $\varepsilon$ simulations. However, more research should be done to study the phenomenon of enlargement and oscillations of the bubbles plume, which is very important in the mixing process in the column. Perhaps, study of the effect of turbulence kinetic energy source on the velocity fluctuations could emphasize the understanding of the behavior of the bubbles plume, and thus help in better prediction of the enlargement.

\section{ACKNOWLEDGMENTS}

I would like to thank the international mobility program of the Rhône-Alpes region (CMIRA), French that supported my work.

\section{REFERENCES}

Besbes, S., M. El Hajem, H. Ben Aissia, J. Y. Champagne and J. Jay (2015) PIV measurements and Euleri-an-Lagrangian simulations of the unsteady gas-liquid flow in a needle sparger rectangular bubble column.
Chemical Engineering Science 126, 560-572.

Chang, K. C., W. D. Hsieh and C. S, Chen (1995). A modified low-Reynolds number model applicable to re-circulating flow in pipe expansion. Journal of Fluids Engineering Transactions of ASME 117, 417-423.

Chen, R. C., J. Reese and L. S. Fan (1994). Flow structure in a three-dimensional bubble column and three phase-fluidized bed. AIChE Journal 40, 1093-1104.

Cotton, M. A. and J. D. Jackson (1990). Vertical tube airflows in the turbulent mixed convection regime calculated using a lowReynolds number k-e model. International Journal of Heat Mass Transfer 33, 275-286.

Du Cluzeau, A., G. Bois and A. Toutant (2019). Analysis and modelling of Reynolds stresses in turbulent bubbly up-flows from direct numerical simulations. Journal of Fluid Mechanics 866, 132-168.

Ekambara, K. and M. T. Dhotre (2010). CFD simulation of bubble column. Nuclear Engineering and Design 240, 963-969.

Fu, S., Y. Guo, W. Qlan and C. Wang (2003). Recent progress in nonlinear eddy-viscosity turbulence modelling. Acta Mechanica Sinica 19(5): 409-4019

Gorji S, M. Seddighi, C. Ariyaratne, A. E. Vardy, T. O. Donoghued, D. Pokrajac and S. He (2014). A compar-ative study of turbulence models in a transient channel flow. Computers \& Fluids 89, 111-123.

Hinze, J. O. (1975). Turbulence. McGraw-Hill Publishing Co., New York,

Hrenya, C. M., E. J. Bolio, D. Chakrabarti and J. L. Sinclair (1995). Comparison of low Reynolds number $\mathrm{k}$-e turbulence models in predicting fully developed pipe flow. Chemical Engineering Science 50, 1923-1941.

Kawamura, T. and Y. Kodama (2002). Numerical simulation method to resolve interactions 
S. Besbes et al. / JAFM, Vol. 14, No. 1, pp. 1-10, 2021.

between bubbles and turbulence. International Journal of Heat Fluid Flow, 23, 627-638.

Launder, B. E. and D. B. Spalding (1972). Turbulence models and their application to the prediction of internal flows [J]. Heat and Fluid Flow 2, 1972.

Launder, B. E. and D. B. Spalding (1974). The numerical computation of turbulent flows [J]. Computer Methods in Applied Mechanics and Engineering 3,269-89.

Lixing, Z., L. Rongxian and D. Ruxu (2006). Numerical simulation of the effect of void fraction and inlet velocity on two-phase turbulence in bubble-liquid flows. Acta Mechanica Sinica 22,425-432.

Ma, T., C. Santarelli, T. Ziegenhein, D. Lucas and J. Fröhlich (2017). Direct numerical simulation-based Reynolds-averaged closure for bubble-induced turbulence. Physical Review Fluids 2, 034301.

Ma, T., L. Dirk, J. Suad and F. Jochen (2020). Progress in the second-moment closure for bubbly flow based on direct numerical simulation data. Journal of Fluid Mechanics 883, A9.

Mandar, V. T, A. R. Swarnendu and B. J. Jyeshtharaj (2008). CFD simulation of bubble column-An analysis of interphase forces and turbulence models. Chemical Engineering Journal 139, 589-614.

Mathur. A. and S. He (2013). Performance and implementation of the Launder-Sharma lowReynolds number turbulence model. Computers \& Fluids 79, 134-139. Rathore SK, Das MK.

Morsi, S. A. and A. J. Alexander (1972). An investigation of particle trajectories in twophase systems. Journal of Fluid Mechanics $55,193-208$.

Patel, V. C., W. Rodi and G. Scheuerer (1985). Turbulence models for near-wall and lowReynolds number flows, a review. AIAA 23, 1308-1319.

Rathore, S. K and M. K. Das (2013). Comparison of two low-Reynolds number turbulence models for fluid flow study of wall bounded jets. International Journal of Heat Mass Transfer 61, 365-380.

Roghair, I., J. M. Mercado, M. Van Sint Annaland, H. Kuipers, C. Sun and D. Lohse (2011). Energy spectra and bubble velocity distributions in pseudo-turbulence: Numerical simulations vs. experiments. International Jounal of Multiphase Flow 37, 1093-1098.

Santarelli, C., J. Roussel and J. Fröhlich (2016). Budget analysis of the turbulent kinetic energy for bubbly flow in a vertical channel. Chemical Engineering Journal 141, 46-62.

Silva, M. D, M. D'Ávila and M. Mori (2012). Study of the interfacial forces and turbulence models in a bubble column. Computers \& Chemical Engineering 44, 34-44.

Simonin. C. and P. L. Viollet (1990). Predictions of an Oxygen Droplet Pulverization in a Compressible Subsonic Coflowing Hydrogen Flow. Numerical Methods for Multiphase Flows, FED91, 65-82.

Simonin. O. (1990) Eulerian formulation for particle dispersion in turbulent two-phase flows. In M. Sommerfeld, and P. Wennerberg, fifth workshop on two phase flow predictions, Germany: Erlangen, 156-166.

Sokolichin, A. and G. Eigenberger (1994). Gasliquid flow in bubble columns and loop reactors: Part I. Detailed modelling and numerical simulation. Chemical Engineering Science 49, 5735-5746.

Sokolichin. A. G. Eigenberger, A. Lapin and A. Lubbert (1997). Dynamic numerical simulation of gas-liquid two-phase flows Euler/Euler versus Euler/Lagrange. Chemical Engineering Science 52(4), 611- 626.

Van Sint Annaland, M., N. G. Deen and J. A. M. Kuipers (2005). Numerical simulation of gas bubbles behavior using a three-dimensional volume of fluid method. Chemical Engineering Science 60, 2999-3011.

Zhaoshun, Z. (2002) Modern Turbulence and new challenges. Acta Mechanica Sinica 18(4), 309. 327. 